\title{
Correspondence
}

\section{Fulminating encephalopathy and Shigella flexneri infection}

Sir,

There was a disappointing lack of epidemiological information in the paper by Sandyk and Brennan. ${ }^{1}$ It would for example have been interesting to know whether the 3 children reported with fulminating encephalopathy associated with Shigella flexneri infection had acquired the organism from the same source and whether the type 2a strain is unusual in Johannesburg.

A dysenteric encephalopathy in which there is sudden onset of altered consciousness and convulsions, often without signs of colitis initially, has been described among Japanese children. The condition is called Ekiri (Eki means epidemic, ri means diarrhoea) and it used to be a common cause of death in 2-5 year olds in Japan, although it has disappeared in recent years. ${ }^{2}$

Sandyk and Brennan comment that in their patients, organs other than the brain and bowel appeared normal at autopsy. In Ekiri, however, the pathological findings are similar to those described in Reye's Syndrome (RS), with microvesicular fatty infiltration of the liver. Further more, Japanese workers describe an experimental model of RS using Shigella flexneri endotoxin in rabbits. ${ }^{2}$

Enteroinvasive Escherichia coli are microbiologically almost indistinguishable from shigellas and cause identical diarrhoeal disease, although neurological complications have not been described. It is interesting to speculate on the possible role in the aetiology of encephalopathies of unknown cause, of neurotoxins elaborated by some gut organism perhaps as yet unidentified or currently considered non-pathogenic.

\section{References \\ 1 Sandyk R, Brennan MJW. Fulminating encephalopathy associated with Shigella flexneri infection. Arch Dis Child 1983; 58: 70-1. \\ 2 Yamashita F, Yamamoto M, Okada S, Yoshida I, Yoshino M. Reye's Syndrome in Japan. In: JFS Crocker ed. Reye's Syndrome II. New York: Grune and Stratton, 1979: 51-67.}

Susan M Hall

PHLS Communicable Disease Surveillance Centre, 61 Colindale Avenue, London NW9 $5 E Q$

Dr Sandyk and Dr Brennan comment:

The dysenteric encephalopathy described in our 3 cases had been acquired simultaneously in different areas of Johannesburg. All subjects were white and all were admitted to hospital in the same week. Although the type 2a strain appears to be uncommon in the area of Johannesburg, a similar dysenteric encephalopathy was observed in 1976 in Johannesburg. We were, however, unable to find any documentation of these cases.

The shigella encephalopathy described in our cases may have some clinical and pathological resemblance to Reye's syndrome (both characterised by cerebral oedema and lack of cellular infiltration). However, whereas in the latter disorder the primary brunt of the process is believed to be upon the liver (the encephalopathy being mainly secondary to hepatic insufficiency) in our cases there was no pathological and biochemical evidence for hepatic failure or other important metabolic derangement. This would suggest that the dysenteric encephalopathy resulted from a direct neurotoxic effect elaborated by the shigella organism. Whether other neurotoxins perhaps elaborated by some other yet unidentified organisms may have contributed to the fulminating encephalopathy remains speculative.

\section{Birthweight of Asian babies in Birmingham}

Sir,

I read the paper by Clarson et al. ${ }^{1}$ from the Sorrento Hospital in Birmingham with both interest and a sense of disappointment. I agree entirely with the authors' conclusions that (a) no such entity as an 'Asian' standard exists and (b) the details of the subethnic structure of an immigrant population need careful assessment. I was dismayed however to find that their 1978 study lumped 'Pakistani' and 'Bangladeshi' mothers together and compared them to 'Indian' mothers. Political considerations aside, this is not acceptable since the ethnic differences between 'Pakistani' mothers and 'Bangladeshi' mothers are significant. As presently constituted, 'Pakistani' mothers should include Panjabi, Sindhi, Baluchis, and Pathan mothers, who would have more in common genetically and otherwise with their Panjabi (Indian) counterparts across the border. Similarly, 'Bangladeshi' mothers would have more in common (genetically and otherwise) with their counterparts from West Bengal (India).

The size and complexity of the Indian subcontinent needs to be emphasized, and a simple reference to an atlas would indicate that the Indian subcontinent should be compared to the whole of western Europe in terms of both geography and population. Religious differences and eating taboos also complicate the picture. Furthermore, in the case of the 'Asian' populations studied what proportion of the mothers in both 'Pakistani' and 'Indian' groups were 'East African Asians'? Were all mothers of the Islamic faith characterised as 'Pakistanis' and all Hindus/Sikhs classified as 'Indian'?

I believe the secular changes in birthweight outlined by Clarson et al. are of great interest, but that more attention should have been paid to the ethnic and cultural details. This would mean that a much larger series would have to be collected and studied, but the importance of the study would justify this. 